\title{
ON THE INTEGRABILITY OF A MUTHUSWAMY-CHUA SYSTEM
}

\author{
JAUME LLIBRE \\ Departament de Matemàtiques, Universitat Autònoma de Barcelona \\ 08193 Bellaterra, Barcelona, Catalonia, Spain \\ jllibre@mat.uab.cat \\ CLAUDIA VALLS \\ Departamento de Matemática, Instituto Superior Técnico \\ Universidade Técnica de Lisoba \\ Av. Rovisco Pais 1049-001, Lisboa, Portugal \\ cvalls@math.ist.utl.pt
}

Received 18 April 2012

Accepted 28 June 2012

Published 31 December 2012

In this paper we study the integrability of the Muthuswamy-Chua system

$$
x^{\prime}=y, \quad y^{\prime}=-\frac{x}{3}+\frac{y}{2}-\frac{y z^{2}}{2}, \quad z^{\prime}=y-\alpha z-y z .
$$

For $\alpha=0$ we characterize all its generalized rational first integrals, which contains the Darboux type first integrals. For $\alpha \neq 0$ we show that the system has no Darboux type first integrals.

Keywords: Darboux integrability; exponential factor; Darboux polynomials; Chua system; generalized rational first integrals.

Mathematics Subject Classification 1991: 34C05, 34A34

\section{Introduction}

Muthuswamy and Chua in [9] proposed the simplest electronic circuit producing chaotic attractors. This circuit contains a linear passive inductor, a linear passive capacitor and a nonlinear active memristor, and it can be described by the differential system

$$
\left\{\begin{array}{l}
x^{\prime}=y, \\
y^{\prime}=-\frac{x}{3}+\frac{y}{2}-\frac{y z^{2}}{2}, \\
z^{\prime}=y-\alpha z-y z
\end{array}\right.
$$

for any value of $\alpha \in \mathbb{R}$, where the prime denotes derivative with respect to the variable $t$. Here, as in [6], this differential system is written in a slightly modified form because the third equation is not $z^{\prime}=-y-\alpha z+y z$ as in [9] but rather $z^{\prime}=y-\alpha z-y z$. The nonlinearity 
is inverted and thus the orientation of the attractor in the $(x, y)$ plane is rotated by $\pi$. Hence there is no topologically difference between these two attractors. A topological characterization of this system and its comparison with the Rössler-like attractors was given in [6].

We define the vector field $X$ associated to (1) as

$$
X=y \frac{\partial}{\partial x}+\left(-\frac{x}{3}+\frac{y}{2}-\frac{y z^{2}}{2}\right) \frac{\partial}{\partial y}+(y-\alpha z-y z) \frac{\partial}{\partial z} .
$$

Let $U$ be an open subset in $\mathbb{R}^{3}$ such that $\mathbb{R}^{3} \backslash U$ has zero Lebesgue measure. We say that a nonconstant real function $H=H(x, y, z): \mathbb{R}^{3} \rightarrow \mathbb{R}$ is a first integral if $H(x(t), y(t), z(t))$ is constant for all values of a solution $(x(t), y(t), z(t))$ of $X$ contained in $U$, i.e. $X H_{\mid U}=0$.

The existence of a first integral for a differential system in $\mathbb{R}^{3}$ allows to reduce its study in one dimension. This is the main reason to look for first integrals.

Two functions $f_{1}(x, y, z)$ and $f_{2}(x, y, z)$ are said to be independent if their gradients are linearly independent vectors for all $(x, y, z) \in \mathbb{R}^{3}$ except perhaps for a set of zero Lebesgue measure. If the vector field $X$ has two independent first integrals $H_{1}$ and $H_{2}$, we say that it is completely integrable. In this case, the orbits of $X$ are contained in the curves $\left\{H_{1}(x, y, z)=h_{1}\right\} \cap\left\{H_{2}(x, y, z)=h_{2}\right\}$, where $h_{1}, h_{2}$ vary in $\mathbb{R}$.

We define a generalized rational function as the quotient of two analytic functions. The following is our main result for system (1) with $\alpha=0$.

Theorem 1. The unique generalized rational first integrals of system (1) with $\alpha=0$ are generalized rational functions in the variable $e^{x}(1-z)$.

Theorem 1 is proved in Sec. 2.

Now we work with $\alpha \neq 0$. One of the best tools to look for first integrals is the Darboux theory of integrability. Now we shall introduce its basic notions. Let $\mathbb{C}[x, y, z]$ be the ring of all polynomials with coefficients in $\mathbb{C}$.

We say that $f \in \mathbb{C}[x, y, z]$ is a Darboux polynomial of the vector field $X$ if there exists a polynomial $k \in \mathbb{C}[x, y, z]$ such that $X f=k f$. The polynomial $k=k(x, y, z)$ is called the cofactor of $f$. It is easy to show that the cofactor of a Darboux polynomial of system (1) has degree at most 2. Note that we look for complex Darboux polynomials in real differential systems. The reason is that frequently the complex structure forces the existence of real first integrals, and sometimes if we only work with reals we cannot detect all the real first integrals.

If $f \in \mathbb{C}[x, y, z]$ is a Darboux polynomial, then $f(x, y, z)=0$ is an invariant algebraic surface for the differential system (1), i.e. if an orbit has a point on the surface $f(x, y, z)=0$ all the orbit is contained in it. See [1] and the references quoted there, where the Darboux polynomials for the Lotka-Volterra systems have been studied.

An exponential factor $F(x, y, z)$ of the vector field $X$ is an exponential function of the form $\exp (g / h)$ with $g$ and $h$ coprime polynomials in $\mathbb{C}[x, y, z]$ and satisfying $X F=L F$ for some $L \in \mathbb{C}[x, y, z]$ with degree at most 2. The exponential factors appear when some Darboux polynomial has multiplicity larger than one, for more details see [3, 7].

A first integral of system (1) is called Darboux type if it is a first integral of the form

$$
f_{1}^{\lambda_{1}} \cdots f_{p}^{\lambda_{p}} F_{1}^{\mu_{1}} \cdots F_{q}^{\mu_{q}}
$$

where $f_{1}, \ldots, f_{p}$ are Darboux polynomials and $F_{1}, \ldots, F_{q}$ are exponential factors. 
The next theorem is the main result for system (1) with $\alpha \neq 0$, and it shows the nonexistence of first integrals of Darboux type.

Theorem 2. When $\alpha \neq 0$ system (1) has no first integrals of Darboux type.

Theorem 2 is proved in Sec. 4.

\section{Proof of Theorem 1}

To prove Theorem 1 we use the following result which is due to Llibre and Zhang in [4].

Theorem 3. Assume that the differential system (1) has $p$ as a singular point and let $\lambda_{1}, \lambda_{2}, \lambda_{3}$ be the eigenvalues of the linear part of system (1) at $p$. Then the number of functionally independent generalized rational first integrals of system (1) is at most the dimension of the minimal vector subspace of $\mathbb{R}^{3}$ containing the set

$$
\left\{\left(k_{1}, k_{2}, k_{3}\right) \in \mathbb{Z}^{3}: k_{1} \lambda_{1}+k_{2} \lambda_{2}+k_{3} \lambda_{3}=0,\left(k_{1}, k_{2}, k_{3}\right) \neq(0,0,0)\right\} .
$$

Proof of Theorem 1. We consider system (1) with $\alpha=0$, that is

$$
\dot{x}=y, \quad \dot{y}=-\frac{x}{3}+\frac{y}{2}-\frac{y z^{2}}{2}, \quad \dot{z}=y-y z .
$$

It is easy to check that

$$
H=x+\ln (1-z)
$$

is a first integral of system (2). Therefore, $K=e^{H}=e^{x}(1-z)$ is a generalized rational function. To conclude the proof of the theorem we shall show that system (2) has no other generalized rational first integrals. To prove this we will use Theorem 3 . First we note that the singular points of system $(2)$ are of the form $(0,0, z)$ with $z \in \mathbb{R}$. We compute the eigenvalues $\lambda_{1}, \lambda_{2}, \lambda_{3}$ of the Jacobian matrix of system (2) on these singular points and we get

$$
\lambda_{1}=0, \quad \lambda_{2,3}=\frac{1}{12}\left(3-3 z^{2} \mp \sqrt{-39+9 z^{2}\left(z^{2}-2\right)}\right) .
$$

Therefore $k_{1} \lambda_{1}+k_{2} \lambda_{2}+k_{3} \lambda_{3}=0$ is equivalent to

$$
k_{2}\left(3-3 z^{2}-\sqrt{-39+9 z^{2}\left(z^{2}-2\right)}\right)+k_{3}\left(3-3 z^{2}+\sqrt{-39+9 z^{2}\left(z^{2}-2\right)}\right)=0,
$$

or in other words

$$
\frac{k_{2}}{k_{3}}=-\frac{3-3 z^{2}+\sqrt{-39+9 z^{2}\left(z^{2}-2\right)}}{3-3 z^{2}-\sqrt{-39+9 z^{2}\left(z^{2}-2\right)}} .
$$

It is clear that the left-hand side of $(3)$ is a rational number (once that $k_{2}, k_{3} \in \mathbb{Z}$ ), and that choosing $z$ in a convenient way the right-hand side of (3) is irrational. Therefore (3) cannot hold for this convenient choice of $z$. Hence for this special singular point $(0,0, z)$, 
the dimension of the minimal vector subspace of $\mathbb{R}^{3}$ containing the set

$$
\left\{\left(k_{1}, k_{2}, k_{3}\right) \in \mathbb{Z}^{3}: k_{1} \lambda_{1}+k_{2} \lambda_{2}+k_{3} \lambda_{3}=0,\left(k_{1}, k_{2}, k_{3}\right) \neq(0,0,0)\right\}
$$

is clearly one, generated by $\left(k_{1}, 0,0\right)$. Thus it follows from Theorem 3 that system $(2)$ can only have one independent generalized rational first integral, which must be a function of $H$. This completes the proof of the theorem.

\section{Auxiliary Results}

In the proof of Theorem 2 we will use the following well known result of the Darboux theory of integrability, see for instance [5, Chap. 8].

Theorem 4 (Darboux theory of integrability). Suppose that a polynomial vector field $X$ defined in $\mathbb{R}^{n}$ of degree $m$ admits $p$ Darboux polynomials $f_{i}$ with cofactors $K_{i}$ for $i=$ $1, \ldots, p$, and $q$ exponential factors $F_{j}=\exp \left(g_{j} / h_{j}\right)$ with cofactors $L_{j}$ for $j=1, \ldots, q$. If there exist $\lambda_{i}, \mu_{j} \in \mathbb{C}$ not all zero such that

$$
\sum_{i=1}^{p} \lambda_{i} K_{i}+\sum_{j=1}^{q} \mu_{j} L_{j}=0,
$$

then the following real (multivalued) function of Darboux type

$$
f_{1}^{\lambda_{1}} \cdots f_{p}^{\lambda_{p}} F_{1}^{\mu_{1}} \cdots F_{q}^{\mu_{q}}
$$

substituting $f_{i}^{\lambda_{i}}$ by $\left|f_{i}\right|^{\lambda_{i}}$ if $\lambda_{i} \in \mathbb{R}$ is a first integral of the vector field $X$.

For a proof of the next result see $[7,8]$.

Proposition 5. The following statements hold.

(a) If $e^{g / h}$ is an exponential factor for the polynomial differential system (1) and $h$ is not a constant polynomial, then $h=0$ is an invariant algebraic surface.

(b) Eventually $e^{g}$ can be an exponential factor, coming from the multiplicity of the infinite invariant plane.

The proof of the next result can be found in [2].

Lemma 6. Let $f$ be a polynomial and $f=\prod_{j=1}^{s} f_{j}^{\alpha_{j}}$ be its decomposition into irreducible factors in $\mathbb{C}[x, y, z]$. Then $f$ is a Darboux polynomial of system (1) if and only if all the $f_{j}$ are Darboux polynomials of system (1). Moreover, if $K$ and $K_{j}$ are the cofactors of $f$ and $f_{j}$, then $K=\sum_{j=1}^{s} \alpha_{j} K_{j}$.

We note that in view of Lemma 6 to study the Darboux polynomials of system (1) it is enough to study the irreducible ones.

\section{Proof of Theorem 2}

To prove Theorem 2 we first study the existence of polynomial first integrals.

Proposition 7. System (1) has no polynomial first integrals. 
Proof. Let $F=F(x, y, z)$ be a polynomial first integral of system (1). Then it satisfies

$$
y \frac{\partial F}{\partial x}+\left(-\frac{x}{3}+\frac{y}{2}-\frac{y z^{2}}{2}\right) \frac{\partial F}{\partial y}+(y-\alpha z-y z) \frac{\partial F}{\partial z}=0 .
$$

We write $F$ as powers in the variable $z$ in the form $F(x, y, z)=\sum_{j=0}^{n} F_{j}(x, y) z^{j}$, where each $F_{j}$ is a polynomial in the variables $x, y$. Computing the coefficient in (6) of $z^{n+2}$ we obtain

$$
-\frac{y}{2} \frac{\partial F_{n}}{\partial y}=0 \quad \text { that is } F_{n}=F_{n}(x)
$$

Now, computing the coefficient in (6) of $z^{n+1}$ we obtain

$$
-\frac{y}{2} \frac{\partial F_{n-1}}{\partial y}=0 \quad \text { that is } F_{n-1}=F_{n-1}(x) .
$$

Finally, computing the coefficient in (6) of $z^{n}$ we get

$$
-\frac{y}{2} \frac{\partial F_{n-2}}{\partial y}+y \frac{d F_{n}}{d x}-(\alpha+y) n F_{n}=0 .
$$

That is, if $n \neq 0, F_{n}=F_{n}(x)$ must be divisible by $y$, in contradiction with the fact that $F_{n}$ is a polynomial in the variable $x$. Therefore, for $n \neq 0$ we must have $F_{n}=0$. Thus, $F=F_{0}(x, y)$. Now, computing the coefficient in (6) of $z^{2}$ we obtain

$$
-\frac{y}{2} \frac{\partial F_{0}}{\partial y}=0 \text { that is } F_{0}=F_{0}(x)
$$

and again by (6) we obtain

$$
y \frac{d F_{0}}{d x}=0 \quad \text { that is } F_{0}=C_{0} \in \mathbb{R}
$$

in contradiction with the fact that $F$ is a polynomial first integral of system (1). This concludes the proof of the proposition.

Now we will study the existence of Darboux polynomials with nonzero cofactor. Note that the Darboux polynomial with zero cofactor is the polynomial first integrals. We recall that a Darboux polynomial of system (1) is a non-constant polynomial $f \in \mathbb{C}[x, y, z]$ such that

$$
y \frac{\partial f}{\partial x}+\left(-\frac{x}{3}+\frac{y}{2}-\frac{y z^{2}}{2}\right) \frac{\partial f}{\partial y}+(y-\alpha z-y z) \frac{\partial f}{\partial z}=k f,
$$

for some polynomial $k \in \mathbb{C}[x, y, z]$ called the cofactor. Since the degree of system $(1)$ is three we have that the degree of $k$ can be at most two, that is, $k$ is of the form

$$
k=a_{0}+a_{1} x+a_{2} y+a_{3} z+a_{4} x^{2}+a_{5} x y+a_{6} x z+a_{7} y^{2}+a_{8} y z+a_{9} z^{2},
$$

where $a_{i} \in \mathbb{C}$ for $i=0, \ldots, 9$.

Proposition 8. System (1) has no Darboux polynomials with nonzero cofactor. 
Proof. Let $f$ be a Darboux polynomial of system (1) with cofactor $k$ given in (8). First we claim that $a_{1}=a_{3}=a_{4}=a_{5}=a_{6}=a_{7}=a_{8}=0$ and $a_{9}=-m / 2$, where $m$ is a non-negative integer.

Now we prove the claim. We write $f$ as sum of its homogeneous parts as follows $f=$ $\sum_{j=0}^{n} f_{j}(x, y, z)$, where each $f_{j}$ is a homogeneous polynomial in its variables. Without loss of generality we can assume that $f_{n} \neq 0$ and $n \geq 1$. Computing the terms of degree $n+2$ in (7) we have

$$
-\frac{y}{2} z^{2} \frac{\partial f_{n}}{\partial y}=\left(a_{4} x^{2}+a_{5} x y+a_{6} x z+a_{7} y^{2}+a_{8} y z+a_{9} z^{2}\right) f_{n}
$$

The general solution of this linear differential equation is of the form

$$
f_{n}=K_{n}(x, z) \exp \left(-\frac{2 a_{5} x}{z^{2}} y-\frac{2 a_{8}}{z} y-\frac{a_{7}}{z^{2}} y^{2}\right) y^{-\frac{2 a_{4} x^{2}}{z^{2}}-\frac{2 a_{6} x}{z}} y^{-2 a_{9}},
$$

where $K_{n}$ is an arbitrary function in the variables $x, z$. Since $f_{n}$ must be a polynomial we get that $a_{4}=a_{5}=a_{6}=a_{7}=a_{8}=0$ and $a_{9}=-m / 2$, where $m$ is a non-negative integer. Therefore

$$
f_{n}=K_{n} y^{m}, \quad \text { where } K_{n}=K_{n}(x, z) \text { is an arbitrary polynomial in } x, z .
$$

Now computing the terms of degree $n+1$ in (7) we obtain

$$
-\frac{y}{2} z^{2} \frac{\partial f_{n-1}}{\partial y}-y z \frac{\partial f_{n}}{\partial z}=-\frac{m}{2} z^{2} f_{n-1}+\left(a_{1} x+a_{2} y+a_{3} z\right) f_{n}
$$

that is

$$
-\frac{y}{2} z^{2} \frac{\partial f_{n-1}}{\partial y}-y z \frac{d K_{n}}{d z} y^{m}=-\frac{m}{2} z^{2} f_{n-1}+\left(a_{1} x+a_{2} y+a_{3} z\right) K_{n} y^{m} .
$$

Solving this linear differential equation we obtain

$$
f_{n-1}=K_{n-1} y^{m}-\frac{2 y^{m}}{z^{2}}\left(a_{2} K_{n} y+\frac{d K_{n}}{d z} y+\left(a_{1} x+a_{3} z\right) K_{n} \log y\right),
$$

where $K_{n-1}=K_{n-1}(x, z)$ is an arbitrary function in $x, z$. Since $f_{n-1}$ must be a polynomial and $K_{n} \neq 0$ (otherwise $f_{n}=0$ ) we conclude that $a_{1}=a_{3}=0$. Hence, the claim is proved.

Now we make the change of variables

$$
x=X, \quad y=\mu^{-2} Y, \quad z=\mu^{-1} Z, \quad t=\mu^{2} \tau,
$$

then the Muthuswamy-Chua system becomes

$$
\dot{X}=Y, \quad \dot{Y}=-\frac{Y Z^{2}}{2}+\mu^{2} \frac{Y}{2}-\mu^{4} \frac{X}{3}, \quad \dot{Z}=-Y Z+\mu Y-\alpha \mu^{2} Z,
$$

where the dot denotes derivative with respect to the variable $\tau$. Set $F(X, Y, Z)=$ $\mu^{n} f\left(X, \mu^{-2} Y, \mu^{-1} Z\right)$ and $K(X, Y, Z)=\mu^{2} k\left(X, \mu^{-2} Y, \mu^{-1} Z\right)$, whereas we considered above 
$n$ is the highest weight degree in the weight homogeneous components of $f$ in the variables $(x, y, z)$ with weight degrees $(0,2,1)$. Assume that

$$
F=\sum_{i=0}^{n} \mu^{i} F_{i}(X, Y, Z)
$$

where $F_{i}$ is a weight homogenous polynomial in $X, Y$ and $Z$ with weight degree $n-i$ for $i=0,1, \ldots, n$. Note that in particular

$$
F_{j}(X, Y, Z)=\mu^{n} f_{n-j}(x, y, z), \quad j=0, \ldots, n .
$$

From the definition of Darboux polynomial we have that

$$
\begin{aligned}
& Y \sum_{i=0}^{n} \mu^{i} \frac{\partial F_{i}}{\partial X}+\left(-\frac{Y Z^{2}}{2}+\mu^{2} \frac{Y}{2}-\mu^{4} \frac{X}{3}\right) \sum_{i=0}^{n} \mu^{i} \frac{\partial F_{i}}{\partial Y} \\
& \quad+\left(-Y Z+\mu Y-\alpha \mu^{2} Z\right) \sum_{i=0}^{n} \mu^{i} \frac{\partial F_{i}}{\partial Z}=\left(a_{2} Y-\frac{m}{2} Z^{2}+\mu^{2} a_{0}\right) \sum_{i=0}^{n} \mu^{i} F_{i} .
\end{aligned}
$$

Equating the terms with $\mu^{i}$ for $i=0,1$ we get

$$
L\left[F_{0}\right]=\left(a_{2} Y-\frac{m}{2} Z^{2}\right) F_{0} \quad \text { and } \quad L\left[F_{1}\right]=\left(a_{2} Y-\frac{m}{2} Z^{2}\right) F_{1}-Y \frac{\partial F_{0}}{\partial Z},
$$

where $L$ is the linear partial differential operator of the form

$$
L=Y \frac{\partial}{\partial X}-\frac{1}{2} Y Z^{2} \frac{\partial}{\partial Y}-Y Z \frac{\partial}{\partial Z}
$$

The characteristic equations associated to the linear partial differential operator are

$$
\frac{d X}{d Z}=-\frac{Y}{Y Z}=-\frac{1}{Z}, \quad \frac{d Y}{d Z}=\frac{Y Z^{2}}{2 Y Z}=\frac{Z}{2}
$$

This system has the general solution

$$
e_{1}=Z e^{X} \quad \text { and } \quad e_{2}=Y-\frac{Z^{2}}{4}
$$

where $e_{1}$ and $e_{2}$ are constants of integration. According to this, we introduce the change of variables

$$
u=Z e^{X}, \quad v=Y-\frac{Z^{2}}{4}, \quad w=X
$$

Its inverse transformation is

$$
Z=u e^{-w}, \quad Y=v+\frac{u^{2} e^{-2 w}}{4}, \quad X=w .
$$

Under the changes (14) and (15), the first equation in (13) becomes the following ordinary differential equation (for fixed $u, v$ ):

$$
\left(v+\frac{u^{2} e^{-2 w}}{4}\right) \frac{\partial \bar{F}_{0}}{\partial w}=\left(a_{2}\left(v+\frac{v^{2} e^{-2 w}}{4}\right)-\frac{m}{2} u^{2} e^{-2 w}\right) \bar{F}_{0},
$$




\section{J. Llibre \& C. Valls}

where $\bar{F}_{0}$ is $F_{0}$ written in terms of $u, v$ and $w$. In what follows we always use the notation $\bar{G}$ to denote $G(x, y, z)$ written in terms of $u, v, w$. The last equation has the general solution

$$
\bar{F}_{0}=\bar{K}_{0}(u, v) e^{a_{2} w}\left(v+\frac{u^{2} e^{-2 w}}{4}\right)^{m},
$$

where $\bar{K}_{0}$ is an arbitrary smooth function in $u$ and $v$. So,

$$
F_{0}=\bar{F}_{0}=e^{a_{2} X} Y^{m} K_{0}\left(Z e^{X}, Y-\frac{Z^{2}}{4}\right) .
$$

Since $F_{0}$ must be a weight homogeneous polynomial of weight degree $n$, we must have $a_{2}=-l$ for some non-negative integer $l$ and

$$
F_{0}=c_{0} Z^{l} Y^{m} P_{0}\left(Y-\frac{Z^{2}}{4}\right)
$$

where $P_{0}$ is a polynomial in the variable $Y-Z^{2} / 4$ and $c_{0} \in \mathbb{C} \backslash\{0\}$. Since the weight degree of $Y-Z^{2} / 4$ is 2 , we get that

$$
F_{0}=c_{0} Z^{l} Y^{m}\left(Y-\frac{Z^{2}}{4}\right)^{p}, \quad l+2 m+2 p=n .
$$

Now from (9), (12) and (16) we obtain

$$
p=0, \quad K_{n}=c_{0} z^{l} \quad \text { and } \quad f_{n}=c_{0} z^{l} y^{m}, \quad F_{0}=c_{0} Z^{l} Y^{m} .
$$

Note that from (10) we get that

$$
f_{n-1}=K_{n-1} y^{m}-\frac{2 l c_{0} y^{m+1}}{z^{2}}\left(-z^{l}+z^{l-1}\right) .
$$

Now, substituting $F_{0}$ into the second equation of (13) we get that

$$
L\left[F_{1}\right]=\left(-l Y-\frac{m}{2} Z^{2}\right) F_{1}-c_{0} l z^{l-1} y^{m+1} .
$$

Using again the change of variables (14) and (15) and working as we did for $F_{0}$ we get that

$$
\begin{aligned}
\left(v+\frac{u^{2} e^{-2 w}}{4}\right) \frac{\partial \bar{F}_{1}}{\partial w}= & \left(-l\left(v+\frac{v^{2} e^{-2 w}}{4}\right)-\frac{m}{2} u^{2} e^{-2 w}\right) \bar{F}_{1} \\
& -c_{0} l u^{l-1} e^{-(l+1) w}\left(v+\frac{u^{2} e^{-2 w}}{4}\right)^{m+1} .
\end{aligned}
$$

Integrating this equation with respect to $w$ we get

$$
F_{1}=-c_{0} l Y^{m} Z^{l-1}+e^{-l X} Y^{m} K_{1}\left(Z e^{X}, Y-\frac{Z^{2}}{4}\right) .
$$

Note that since $F_{1}$ must be a polynomial of degree $n-1$, proceeding as we did for $F_{0}$ we have that

$$
e^{-l X} Y^{m} K_{1}\left(Z e^{X}, Y-\frac{Z^{2}}{4}\right)=Y^{m} Z^{l} P_{1}\left(Y-\frac{Z^{2}}{4}\right),
$$

where $P_{1}$ is a polynomial in the variable $Y-Z^{2} / 4$. Note that $F_{1}$ has weight degree $2 m+l+2 q$ where $q$ is the degree of $P_{1}$ with respect to $Y-Z^{2} / 4$. Since $F_{1}$ has degree $2 m+2 l-1$ it 
follows that $P_{1}=0$. Thus

$$
F_{1}=-c_{0} l Y^{m} Z^{l-1}
$$

Now from this equation, Eqs. (12), (17) and the fact that $c_{0} \neq 0$ we get

$$
l=0, \quad K_{n}=c_{0}, \quad f_{n}=c_{0} y^{m}, \quad f_{n-1}=0 .
$$

Therefore, $a_{2}=0$. Now computing the terms of degree $n$ in (7) we obtain

$$
-\frac{y}{2} z^{2} \frac{\partial f_{n-2}}{\partial y}+m\left(-\frac{x}{3}+\frac{y}{2}\right) c_{0} y^{m-1}=-\frac{m}{2} z^{2} f_{n-2}+a_{0} c_{0} y^{m} .
$$

Solving it

$$
f_{n-2}=y^{m} K_{n-2}+\frac{c_{0} y^{m}}{z^{2}}\left(\frac{2 m x}{3 y}+\left(m-2 a_{0}\right) \log y\right),
$$

where $K_{n-2}=K_{n-2}(x, z)$ is an arbitrary function in $x, z$. Since $f_{n-2}$ must be a polynomial and $c_{0} \neq 0$ we get that $m=0$ and $a_{0}=m / 2=0$. Therefore, since $a_{9}=-m / 2$ we conclude that $a_{i}=0$ for $i=0, \ldots, 9$ and thus $k=0$, in contradiction with the fact that $f$ is a Darboux polynomial with nonzero cofactor. This concludes the proof of the proposition.

An exponential factor of system (1) is a function $f=\exp (h / g)$ satisfying (7), where $h, g \in \mathbb{C}[x, y, z]$ are coprime polynomials.

Proposition 9. The unique exponential factors, modulo constants, of system (1) are

$$
e^{x}, \quad e^{x^{2}}, \quad e^{z} \quad \text { and } e^{z^{2}-4 y}
$$

with cofactors

$$
y, \quad 2 x y, \quad y-y z-\alpha z \quad \text { and } \quad \frac{4}{3} x-2 y+2 y z-2 \alpha z^{2},
$$

respectively.

Proof. Let $F=\exp (g / h)$ be an exponential factor of the Muthuswamy-Chua system with cofactor $L$, where $g, h \in \mathbb{C}[x, y, z]$ with $(g, h)=1$. Then from the definition of exponential factor and in view of Propositions 5, 7 and 8 we have that $h$ is a constant that we can take $h=1$. Thus $F=\exp (g)$, where $g \in \mathbb{C}[x, y, z]$. Then, $g$ satisfies

$$
y \frac{\partial g}{\partial x}+\left(-\frac{x}{3}+\frac{y}{2}-\frac{y z^{2}}{2}\right) \frac{\partial g}{\partial y}+(y-\alpha z-y z) \frac{\partial g}{\partial z}=L,
$$

where $L=a_{0}+a_{1} x+a_{2} y+a_{3} z+a_{4} x^{2}+a_{5} x y+a_{6} x z+a_{7} y^{2}+a_{8} y z+a_{9} z^{2}$. We write $g$ as a polynomial in the variable $z$ in the form

$$
g(x, y, z)=\sum_{j=0}^{n} g_{j}(x, y) z^{j}
$$

where each $g_{j}$ is a polynomial in the variables $x, y$. We first assume $n \geq 3$. Computing the coefficient in (18) of $z^{n+2}$ we obtain

$$
-\frac{y}{2} \frac{\partial g_{n}}{\partial y}=0 \quad \text { that is } g_{n}=g_{n}(x) .
$$




\section{J. Llibre \&s C. Valls}

Now computing the coefficient in (18) of $z^{n+1}$ we obtain

$$
-\frac{y}{2} \frac{\partial g_{n-1}}{\partial y}=0 \quad \text { that is } g_{n-1}=g_{n-1}(x)
$$

Finally, computing the coefficient in (18) of $z^{n}$ we get

$$
-\frac{y}{2} \frac{\partial g_{n-2}}{\partial y}+y \frac{d g_{n}}{d x}-(\alpha+y) n g_{n}=0
$$

Then $g_{n}=g_{n}(x)$ must be divisible by $y$, in contradiction with the fact that $g_{n}$ is a polynomial in the variable $x$. Therefore $n<3$ and we write $h$ as $g=g_{0}(x, y)+g_{1}(x, y) z+g_{2}(x, y) z^{2}$, where $g_{i}$ are polynomials in $x, y$ for $i=0,1,2$. Imposing that $g$ satisfies (18) and computing the coefficients of $z^{j}$, that we call $A_{j}$ for $j=0,1,2,3,4$ in (18) we get

$$
\begin{aligned}
& A_{1}=\frac{1}{6}\left(-6 a_{0}-6 a_{1} x-6 a_{4} x^{2}-6 a_{2} y-6 a_{5} x y-6 a_{7} y^{2}+6 y g_{1}+(3 y-2 x) \frac{\partial g_{0}}{\partial y}+6 y \frac{\partial g_{0}}{\partial x}\right) \\
& A_{2}=\frac{1}{6}\left(-6 a_{3}-6 a_{6} x-6 a_{8} y-6(y+\alpha) g_{1}+12 y h_{2}+(3 y-2 x) \frac{\partial g_{1}}{\partial y}+6 y \frac{\partial g_{1}}{\partial x}\right) \\
& A_{3}=\frac{1}{6}\left(-6 a_{9}-12(y+\alpha) g_{2}-3 y \frac{\partial g_{0}}{\partial y}+(3 y-2 x) \frac{\partial g_{2}}{\partial y}+6 y \frac{\partial g_{2}}{\partial x}\right) \\
& A_{4}=-\frac{1}{2} y \frac{\partial g_{1}}{\partial y} \\
& A_{5}=-\frac{1}{2} y \frac{\partial g_{2}}{\partial y}
\end{aligned}
$$

From conditions $A_{4}=A_{5}=0$ we get that $g_{1}(x, y)=g_{1}(x)$ and $g_{2}(x, y)=g_{2}(x)$. Now the equation for $A_{2}$ becomes

$$
A_{2}=-6\left(a_{3}+a_{6} x+\alpha g_{1}\right)-6 y\left(a_{8}+g_{1}-2 g_{2}-\frac{d g_{1}}{d x}\right)
$$

and setting $A_{2}=0$ we obtain

$$
g_{1}=-\frac{1}{\alpha}\left(a_{3}+a_{6} x\right) \quad \text { and } \quad g_{2}=\frac{1}{2 \alpha}\left(a_{8} \alpha+a_{6}-a_{3}-a_{6} x\right) .
$$

Furthermore, condition $A_{3}$ becomes

$$
A_{3}=\frac{1}{2 \alpha}\left(2 a_{3}(y+\alpha)+a_{6}((2 x-3) y+2(x-1) \alpha)-2 \alpha\left(a_{9}+a_{8}(y+\alpha)\right)-y \alpha \frac{\partial g_{0}}{\partial y}\right),
$$

and thus, from $A_{3}=0$ we get

$$
g_{0}=\frac{1}{\alpha}\left(y\left(2 a_{3}+a_{6}(2 x-3)-2 a_{8} \alpha\right)-2 \alpha\left(-a_{3}+a_{6}+a_{9}-a_{6} x+a_{8} \alpha\right) \log y+\alpha G_{0}(x)\right) .
$$

Since $g_{0}$ must be a polynomial we have

$$
a_{6}=0, \quad a_{3}=a_{9}+a_{8} \alpha \quad \text { and } \quad g_{0}=\frac{2 a_{9}}{\alpha} y+G_{0}(x)
$$


Now imposing these conditions into the relation $A_{1}=0$ we obtain

$$
-2 a_{9} x-3\left(a_{0}+a_{1} x+a_{4} x^{2}+y\left(a_{2}+a_{8}+a_{5} x+a_{7} y\right)\right) \alpha+3 y \alpha \frac{d G_{0}}{d x}=0
$$

which yields

$$
G_{0}=K_{0}+\frac{a_{4} x^{3}}{3 y}+\frac{x}{y}\left(a_{0}+\left(a_{2}+a_{8}\right) y+a_{7} y^{2}\right)+\frac{x^{2}}{6 \alpha y}\left(2 a_{9}+3 \alpha a_{1}+3 \alpha a_{5} y\right) .
$$

Since $G_{0}$ must be a polynomial in the variable $x$ we get

$$
a_{4}=0, \quad a_{0}=0, \quad a_{9}=-\frac{3}{2} a_{1} \quad \text { and } \quad a_{7}=0,
$$

and hence $G_{0}=K_{0}+\left(a_{2}+a_{8}\right) x+a_{5} x^{2} / 2$. Moreover, since we are searching the exponential factors modulo constants we can set $K_{0}=0$. In summary

$$
\begin{aligned}
g & =\frac{2 a_{9}}{\alpha} y+\left(a_{2}+a_{8}\right) x+\frac{a_{5}}{2} x^{2}+\left(\frac{3 a_{1}}{2}-a_{8}\right) z+\frac{3 a_{1}}{4} z^{2} \\
& =a_{2} x+\frac{a_{5}}{2} x^{2}+a_{8}(x-z)+a_{1}\left(-3 y+\frac{3 z}{2}+\frac{3 z^{2}}{4}\right) .
\end{aligned}
$$

Note that in modulo multiplicative constants, the exponential factors are $e^{x}, e^{x^{2}}, e^{z}$ and $e^{z^{2}-4 y}$. Knowing the exponential factors we can compute easily their cofactors, obtaining the ones given in the statement of the proposition. This completes the proof of the proposition.

Proof of Theorem 2. It follows from Theorem 4 that the Muthuswamy-Chua system (1) has a first integral of Darboux type if and only if there exist $\lambda_{i}, \mu_{j} \in \mathbb{C}$ not all zero such that Eq. (4) is satisfied, where $p, q$ are the number of Darboux polynomials and the number of exponential factors, respectively. Furthermore, $K_{i}, L_{j}$ are the cofactors of Darboux polynomials and exponential factors, respectively. It follows from Propositions 7 and 8 that the Muthuswamy-Chua system has no Darboux polynomials, and by Proposition 9 we have that there are four cofactors of the form $L_{1}=y, L_{2}=2 x y, L_{3}=y-y z-\alpha z$ and $L_{4}=4 x / 3-2 y+2 y z-2 \alpha z^{2}$. So Eq. (4) is equivalent to

$$
\mu_{1} y+2 \mu_{2} x y+\mu_{3}(y-y z-\alpha z)+\mu_{4}\left(\frac{4}{3} x-2 y+2 y z-2 \alpha z^{2}\right)=0 .
$$

Solving this equation we obtain that $\mu_{1}=\mu_{2}=\mu_{3}=\mu_{4}=0$. This completes the proof of the theorem.

\section{Acknowledgments}

The first author was supported by the grants MICIIN/FEDER MTM 2008-03437, AGAUR 2009SGR410 and ICREA Academia. The second author was (grant PTDC/MAT/ 117106/2010) and supported by FCT through CAMGDS. 


\section{References}

[1] Y. T. Christodoulides and P. A. Damianou, Darboux polynomials for Lotka-Volterra systems in three dimensions, J. Nonlinear Math. Phys. 16 (2009) 339-354.

[2] C. Christopher and J. Llibre, Integrability via invariant algebraic curves for planar polynomial differential systems, Ann. Differential Equations 14 (2000) 5-19.

[3] C. Christopher, J. Llibre and J. V. Pereira, Multiplicity of invariant algebraic curves in polynomial vector fields, Pacific J. Math. 229 (2007) 63-117.

[4] W. Cong, J. Llibre and X. Zhang, Generalized rational first integrals of analytic differential systems, J. Differential Equations 251 (2011) 2770-2788.

[5] F. Dumortier, J. Llibre and J. C. Artés, Qualitative Theory of Planar Differential Systems, Universitext (Springer-Verlag, New York, 2006).

[6] J. Ginoux, C. Letellier and L. O. Chua, Topological analysis of chaotic solution of a three-element memristive circuit, Int. J. Bifurcation Chaos 20 (2010) 3819-3827.

[7] J. Llibre and X. Zhang, Darboux theory of integrability in $\mathbb{C}^{n}$ taking into account the multiplicity, J. Differential Equations 246 (2009) 541-551.

[8] J. Llibre and X. Zhang, Darboux theory of integrability for polynomial vector fields in $\mathbb{R}^{n}$ taking into account the multiplicity at infinity, Bull. Sci. Math. 133 (2009) 765-778.

[9] B. Muthuswamy and L. O. Chua, Simplest chaotic circuit, Int. J. Bifurcation Chaos 20 (2010) $1567-1580$. 